\title{
A Modeling of Multi-Echelon Suppliers' Chain for Deteriorating Items
}

\author{
Jonas Yu ${ }^{1,2}$, G.A. Widyadana ${ }^{3}$
}

\begin{abstract}
Inventory models for a policy of retail pricing and perishable items have been developed in many papers, but very few of the studies incorporate both of these effects. This study integrates the two conditions from a three-echelon supplier's chain and offers a mathematical modeling for deteriorating inventory system with an optimal joint-cost policy. Exponentially deteriorating items with no shortages is assumed in this study. Two scenarios are considered. Scenario 1 assumed one supplier, one distributor and one retailer occurs at the regular inventory replenishment time, and scenario 2 assumes two suppliers, one distributor and two competitive retailers that to address this issue, we broaden the benchmark setting by letting the independent retailer channel be a duopoly. The mathematical model describes how the integrated approach to decision making can achieve global optimality as compared to independent decision by the suppliers, the distributor, and the retailers. A computer code is developed to derive the optimal solution. Numerical examples and sensitivity analysis are given to validate the results of the system.
\end{abstract}

Keywords: Deterioration, supply chain, three-echelon inventory system.

\section{Introduction}

Historically, the three key members of the supply chain, supplier, distributor and retailer, have been managed independently, buffered by large inventories. Increasing competitive pressures and decreesing marginal profitability are forcing firms to develop supply chains that can quickly respond to customer needs and furthermore reduce the cost on carrying inventory. There are three fundamental stages in the supplier chain: Procurement, production and distribution. Through their coordination, the number of deliveries is derived in corporation with each other to achieve a minimum overall integrated cost.

Deterioration is defined as decay, damage, spoilage, evaporation, obsolescence, pilferage, and loss of utility or loss of marginal value of a commodity that results in decreasing usefulness from the original one. Blood, fish, strawberries, alcohol, gasoline, radioactive chemicals and grain products are examples of deteriorating commodities. In this study, deterioration is assumed to be a function of the onhand inventory within the whole supplier chain.

In order to reduce loss due to deterioration of the products, the members of the supply chains fre quently

1 Department of Information Management, Takming College, No.56, Sec.1, Huanshan Rd., Neihu District, Taipei 11451, Taiwan. Email: jonasyu@takming.edu.tw

${ }^{2}$ Institute of Industrial Management, National Central University, Chung-li 32054, Taiwan.

${ }^{3}$ Faculty of Industrial Technology, Department of Industrial Engineering, Petra Christian University, JL. Siwalankerto 121-131 Surabaya 60238, Indonesia. Email: gede@peter.petra.ac.id

Invited Paper. implement a joint decision to the optimal number of deliveries.

Ghare and Schrader [3] were the first authors to consider on-going deterioration of inventory. Then, several researchers have studied deteriorating inventory in recent decades. Bessler and Veinott [2] made the analysis on the integration between the buyer and the supplier by developing a mathematical model with an arborescent structure. Axsater [1] have developed the different models based on the centralized and decentralized echelon. Wee [7] derived the integration model between vendor and buyer for deteriorating items. Rau et al. [5] developed production inventory model for deteriorating items under multi echelon supply chain. Similar model is introduced by Rau et al. [6]. In their model they considered supplier-buyer integrated model by taking account buyer shoratage. Later Lin and Lin [4] developed two-echelon inventory model by considering completed backorder in the problem.

In this paper, a mathematical model taking into account the integration of the producer, distributor and retailer is developed. Since the three players do not have production rate constraints, so the model is different with Rau et al. [5]. Rau et al. [5] considered three players in their model who are supplier, producer and buyer. They assumed that the producer has specific production rate. $\mathrm{Yu}$ [8] proposed an integrated deteriorating inventory model for a multiechelon supply chain with dual source. The numerical example shows that the integrated approach that takes account of the supplier, the distributor and the retailer is better than the independent approach by the individual player. The model has potential application in a supply chain environment where there is a multi-echelon inventory system. This 
three-echelon inventory system usually happens in real life.

\section{Methods}

\section{Mathematical Model}

The mathematical model is developed on the basis of the following assumptions: (a) the demand rate is known and constant; (b) no shortage is allowed; (c) there is no constraint in space, capacity or capital; (d) the rate of replenishment in either distributor or retailers is infinite and on the same time; (e) a constant fraction the on-hand inventory deteriorates and no replacement of deteriorated items is assumed; (f) only a single-product item is considered.

The following notation is used in this study:

$T C$ : total joint cost for supply chain

$T C_{r} \quad$ : total cost for all retailers

$T C_{d} \quad$ : total cost for distributor

$T C_{p} \quad$ : total cost for all suppliers

$Q_{r k}(t)$ : retailer's inventory level at any time $t$, $k=1,2,3$

$Q_{d}(t)$ : distributor's inventory level at any time $t$

$Q_{p i}(t)$ : suppliers's inventory level at any time $t, i=$ 1,2

$C_{r k} \quad$ : ordering cost for retailers, $k=1,2,3$

$C_{d} \quad$ : ordering cost for distributor

$C_{p i} \quad: \quad$ ordering cost for suppliers, $i=1,2$

$H_{r k} \quad$ : carrying cost per unit per unit time for retailers, $k=1,2,3$

$H_{d} \quad$ : carrying cost per unit per unit time for distributor

$H_{p i} \quad$ : carrying cost per unit per unit time for suppliers, $i=1,2$

$P_{r k} \quad:$ deterioration cost per unit per unit time for retailers, $k=1,2,3$

$P_{d} \quad$ : deterioration cost per unit per unit time for distributor

$P_{p i} \quad$ : deterioration cost per unit per unit time for suppliers, $i=1,2$

$d_{i} \quad:$ demand rate for retailers $, k=1,2,3$

$\theta \quad:$ deterioration rate

$m_{i} \quad$ : order arrangement rate, $i=1,2$

$T \quad$ : replenishment cycle time for suppliers

$t_{r i} \quad$ : replenishment cycle time for retailers,$i=$ $1,2,3 \ldots . . n$

$t_{d} \quad$ : replenishment cycle time for distributor

$n_{10} \quad$ : number of deliveries per cycle time $T$ from suppliers to distributor

$n_{0 k} \quad$ : number of deliveries per cycle time $t_{d}$ from distributor to retailers, $k=1,2,3$

As an illustration, the supplier-distributor-retailer inventory system is depicted in Figure 1.

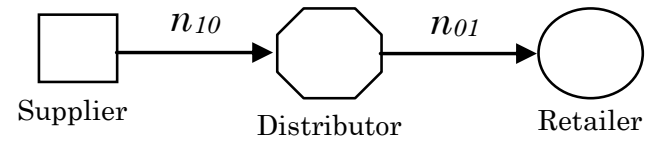

Figure 1. Supplier-distributor-retailer inventory system

We consider the supply chain system consisting of one supplier, one distributor and one retailer, purchasing an item through this chain.

\section{The Retailers' Deteriorating Inventory System}

In general, a retailer's deteriorating inventory system is described as follows:

Retail demand depends on two elements-primary demand for the product and store-level factors that influence consumers' sensitivity to retail. Specifically, let $p$ represent price, and retail demand be $q_{i}=\alpha-\beta p_{i}$ where $\alpha>0$ represents the primary demand and $\beta>0$ represents the store-level factors. The consumers' price decreases proportionally with time going.

$p_{i}=p_{i-1} \lambda, 0<\lambda<1, i=1, \ldots, n$

where $\lambda$ represents the proportional number. The life cycle time of product is $t_{l}$. It means the finished goods should be sold out by the time.

The change inventory level, $d Q_{r i}(t)$, during an infinitesimal time, $d t$, is a function of the deterioration ratea, demand rate $d$ and the inventory level $Q_{r i}(t)$. It is formulated as

$\frac{d Q_{r i}(t)}{d t}=-d-\theta Q_{r i}(t), 0 \leq t \leq t_{r i}$

For a periodic cycle of $t_{r i}$, the inventory level $Q_{r i}\left(t_{r i}\right)=0$ and at the initial time, $Q_{r i}\left(t_{r i}\right)=S_{r i}$; the solution of (1), after adjusting for the constant of integration is

$Q_{r i}(t)=\frac{d_{i}}{\theta}\left[e^{\theta\left(t_{r i}-t\right)}-1\right], 0 \leq t \leq t_{r i}$

From equation (1) and (2), the initial inventory level can be derived as:

$S_{r i}=\frac{-d_{i}+d_{i} e^{\theta t}}{\theta}$

and the deterioration amount during $t_{r i}$ is

$W_{r i}=S_{r i}-d_{i} t_{r i}=\frac{-d_{i}+d_{i} e^{\theta t_{r i}}}{\theta}-d_{i} t_{r i}=A_{r i} \theta$

From equation (4), the carrying inventory during $t_{r i}$ can be derived as:

$A_{r i}=\frac{d_{i} e^{\theta t} r i-d_{i}-\theta d_{i} t_{r i}}{\theta^{2}}$ 
Therefore, the retailer's carrying cost $H C_{r i}$ for a periodic cycle is given by:

$H C_{r i}=A_{r i} \mathrm{x} H_{r}=\left[\frac{d_{i} e^{\theta t} t_{r i-d_{i}-\theta d_{i} t_{r i}}}{\theta^{2}}\right] H_{r}$

The retailers inventory cost per replenishment period $t_{r k}$ is depicted by the following formula: period cost $=$ ordering cost + carrying cost + deteriorating cost

$T C_{r i}=q_{i} c+\frac{d e^{\theta t_{r i-d}-\theta d t_{r i}}}{\theta^{2}} H_{r}+\frac{-d+d e^{\theta t} r i-\theta d t_{r i}}{\theta} P_{r}$

Thus, we obtain the total cost $T C_{r}$ through the production cycle is given by:

$T C_{r}=\sum_{i=1}^{n} T C_{r i}$

$=\sum_{i=1}^{n}\left(q_{i} c+\frac{d e^{\theta t} r i-d-\theta d t_{r i}}{\theta^{2}} H_{r}+\frac{-d+d e^{\theta t_{r i-}-\theta d t_{r i}}}{\theta} P_{r}\right)$

where

$t_{r i}=\frac{q_{r i}}{d}=\frac{\alpha-\beta p_{i}}{d}$

\section{The Distributor's Deteriorating Inventory Sys- tem}

The change inventory level, $d Q_{d}(t)$, during an infinitesimal time, $d t$, is a function of the deterioration ratea, demand rate $d_{k}$ and the inventory level $Q_{d}(t)$. It is formulated as

$\frac{d Q_{d}(t)}{d t}=-\sum_{k=1}^{3} d_{k}-\alpha Q_{d}(t), 0 \leq t \leq t_{d}$

For a periodic cycle of $t_{d}$, the inventory level $Q_{d}\left(t_{d}\right)=0$ and at the initial time, $Q_{d}(0)=S_{d}$; the solution of (11), after adjusting for the constant of integration is

$Q_{d}(t)=\frac{\sum_{k=1}^{3} d_{k}}{\alpha}\left[e^{\alpha\left(t_{d}-t\right)}-1\right], 0 \leq t \leq t_{d}$

From equation (10) and (11), the initial inventory level can be derived as:

$S_{d}=\frac{-\sum_{k=1}^{3} d_{k}+\sum_{k=1}^{3} d_{k} e^{\alpha t} d}{\alpha}$

and the deterioration amount during $t_{d}$ is

$W_{d}=S_{d}-\sum_{k=1}^{3} d_{k} t_{d}-\sum_{k=1}^{3} W_{r k}=A_{d} \alpha$

Thus, the deterioration cost is

$$
\begin{aligned}
& W_{d} \times P_{d}= \\
& {\left[\frac{-\sum_{k=1}^{3} d_{k}+\sum_{k=1}^{3} d_{k} e^{\alpha t_{d}}-\alpha \sum_{k=1}^{3} d_{k} t_{d}}{\alpha}-\right.} \\
& \left.\sum_{k=1}^{3} \frac{-d_{k}+d_{k} e^{\alpha t_{r k}-\alpha d_{k} t_{r k}}}{\alpha}\right] P_{d}
\end{aligned}
$$

From equation (13), the carrying inventory during $t_{d}$ can be derived as:

$A_{d}=$

$\left[\frac{-\sum_{k=1}^{3} d_{k}+\sum_{k=1}^{3} d_{k} e^{\alpha t_{d}}-\alpha \sum_{k=1}^{3} d_{k} t_{d}}{\alpha^{2}}-\right.$

$\left.\sum_{k=1}^{3} \frac{-d_{k}+d_{k} e^{\alpha t_{r k}-\alpha d_{k} t_{r k}}}{\alpha^{2}}\right]$

Thus, the distributor's carrying cost $H C_{d}$ for a periodic cycle is given by:

$H C_{d}=A_{d} \times H_{d}$

Therefore, the total cost for distributor is

$T C_{d}(T)=C_{d} \times n_{10}+H C_{d} \times n_{10}+W_{d} \times n_{10}$

$=C_{d} \times n_{10}+\left[\frac{-\sum_{k=1}^{3} d_{k}+\sum_{k=1}^{3} d_{k} e^{\alpha t} d-\alpha \sum_{k=1}^{3} d_{k} t_{d}}{\alpha^{2}}-\right.$

$\left.\sum_{k=1}^{3} \frac{-d_{k}+d_{k} e^{\alpha t_{r k}-\alpha d_{k} t_{r k}}}{\alpha^{2}}\right] H_{d} \times n_{10}+$

$\left[\frac{-\sum_{k=1}^{3} d_{k}+\sum_{k=1}^{3} d_{k} e^{\alpha t} d-\alpha \sum_{k=1}^{3} d_{k} t_{d}}{\alpha}-\right.$

$\left.\sum_{k=1}^{3} \frac{-d_{k}+d_{k} e^{\alpha t_{r k}-\alpha d_{k} t_{r k}}}{\alpha}\right] P_{d} \times n_{10}$

\section{The Suppliers' Deteriorating Inventory System}

The change inventory level, $d Q_{p i}(t)$, during an infinitesimal time, $d t$, is a function of the deterioration rate $\alpha$, demand rate $d_{k}$, the order arrangement rate $m_{i}$ and the inventory level $Q_{p i}(t)$. It is formulated as

$\frac{d Q_{p i}(t)}{d t}=-\sum_{k=1}^{3} m_{i} d_{k}-\alpha Q_{p i}(t), 0 \leq t \leq T$,

where $\sum_{i=1}^{2} m_{i}=1$

For a periodic cycle of $T$, the inventory level $Q_{p i}(T)=0$ and at the initial time, $Q_{p i}(0)=S_{p i}$; the solution of (18), after adjusting for the constant of integration is

$Q_{p i}(t)=\frac{\sum_{k=1}^{3} m_{i} d_{k}}{\alpha}\left[e^{\alpha(T-t)}-1\right], 0 \leq t \leq T$

From equation (18) and (19), the initial inventory level can be derived as:

$S_{p i}=\frac{-\sum_{k=1}^{3} d_{k} m_{i}+\sum_{k=1}^{3} d_{k} m_{i} e^{\alpha T}}{\alpha}$

and the deterioration quantity during $T$ is

$$
\begin{aligned}
& W_{p i}=S_{p i}-\sum_{k=1}^{3} m_{i} d_{k} T-m_{i} W_{d} \\
& =\frac{-\sum_{k=1}^{3} d_{k} m_{i}+\sum_{k=1}^{3} d_{k} m_{i} e^{\alpha T}}{\alpha}-\sum_{k=1}^{3} m_{i} d_{k} T \\
& -\left(\sum_{k=1}^{3} \frac{-d_{k}+d_{k} e^{\alpha t_{d}-\alpha d_{k} t_{d}}}{\alpha}-\sum_{k=1}^{3} \frac{-d_{k}+d_{k} e^{\alpha t_{r k}-\alpha d_{k} t_{r k}}}{\alpha}-\right) m_{i} \\
& =A_{p i} \alpha
\end{aligned}
$$

Thus, the deterioration cost is 
$W_{p i} \times P_{p i}$

$=\sum_{k=1}^{3}\left[\left(\frac{-d_{k} m_{i}+d_{k} m_{i} e^{\alpha t_{d}-\alpha m_{i} d_{k} t_{d}}}{\alpha}\right)-\left(\frac{-d_{k}+d_{k} e^{\alpha t_{d}-\alpha d_{k} t_{d}}}{\alpha}-\right.\right.$

$\left.\left.\frac{-d_{k}+d_{k} e^{\alpha t_{r k}-\alpha d_{k} t_{r k}}}{\alpha}\right) m_{i}\right] \times P_{p i}$

From equation (21), the carrying inventory within $t_{d}$ can be derived as:

$A_{p i}=$

$\sum_{k=1}^{3}\left[\left(\frac{-d_{k} m_{i}+d_{k} m_{i} e^{\alpha t_{d}-\alpha m_{i} d_{k} t_{d}}}{\alpha^{2}}\right)-\left(\frac{-d_{k}+d_{k} e^{\alpha t_{d}}-\alpha d_{k} t_{d}}{\alpha^{2}}-\right.\right.$

$\left.\left.\frac{-d_{k}+d_{k} e^{\alpha t_{r k}-\alpha d_{k} t_{r k}}}{\alpha^{2}}\right) m_{i}\right]$

Thus, the supplier's carrying cost $H C_{p i}$ for a periodic cycle is given by:

$H C_{p i}=A_{p i} \times H_{p i}$

Thus, the total cost for suppliers is

$T C_{p}(T)=\sum_{i=1}^{2}\left(C_{p i} \times n_{10}+H C_{p i} \times n_{10}+W_{p i} \times n_{10}\right)$

$=$

$\sum_{i=1}^{2} C_{p i} \times n_{10}+\sum_{i=1}^{2} \sum_{k=1}^{3}\left[\left(\frac{-d_{k} m_{i}+d_{k} m_{i} e^{\alpha t_{d}-\alpha m_{i} d_{k} t_{d}}}{\alpha^{2}}\right)-\right.$

$\left.\left(\frac{-d_{k}+d_{k} e^{\alpha t_{d}}-\alpha d_{k} t_{d}}{\alpha^{2}}-\frac{-d_{k}+d_{k} e^{\alpha t_{r k}}-\alpha d_{k} t_{r k}}{\alpha^{2}}\right) m_{i}\right] H_{p i} n_{10}$

$+\sum_{i=1}^{2} \sum_{k=1}^{3}\left[\left(\frac{-d_{k} m_{i}+d_{k} m_{i} e^{\alpha t_{d}-\alpha m_{i} d_{k} t_{d}}}{\alpha}\right)-\right.$

$\left.\left(\frac{-d_{k}+d_{k} e^{\alpha t_{d}-\alpha d_{k} t_{d}}}{\alpha}-\frac{-d_{k}+d_{k} e^{\alpha t_{r k}-\alpha d_{k} t_{r k}}}{\alpha}\right) m_{i}\right] P_{p i} n_{10}$

The Integrated Three-Echelon Deteriorating Inventory System

The integrated total cost can be obtained as:

$T C=T C_{r}+T C_{d}+T C_{p}$

The optimal values of $T, n_{10}, n_{01}, n_{02}$ and $n_{03}$ denoted by $T^{*}, n_{10}^{*}, n_{01}^{*}, n_{02}^{*}$ and $n_{03}^{*}$ respectively, must satisfy equations (27) and (28), and will be located in the vicinity of $n_{0 k}^{\#}$ that satisfy equation (29).

$\frac{d T C}{d T}=0$

$T C\left(n_{i k}^{*}-1\right) \geq T C\left(n_{i k}^{*}\right) \geq T C\left(n_{i k}^{*}+1\right)$

$\frac{d T C}{d n_{i k}^{\#}}=0$

where $i=0,1$ and $k=0,1,2,3$

\section{Result and Discussion}

\section{Numerical Example}

A numerical example is used to illustrate this case discussed. The related data from suppliers are as follows: $T=1$ year, $H_{p 1}=H_{p 2}=\$ 5$ per year, $C_{p 1}=C_{p 2}=\$ 5000$ per time, $P_{p 1}=P_{p 2}=\$ 20$ per unit, $m_{1}=m_{2}=0.5$. The related data from distributor are as follows: $H_{d}=\$ 20$ per unit per year, $C_{d}=\$ 1000$ per time, $P_{d}=\$ 30$ per unit. The related data from retailers are as follows: $d_{1}=d_{2}=10000, d_{3}=6000$, $H_{r 1}=H_{r 2}=\$ 50$ per unit per year, $H_{r 3}=\$ 70$ per unit per year, $C_{r 1}=C_{r 2}=\$ 600$ per time, $C_{r 3}=\$ 600$ per time, $P_{r 1}$ $=P_{r 2}=\$ 50$ per unit, $P_{r 3}=\$ 80$ per unit, $\theta=0.05$. The numerical results are tabulated in Table 1 . The sensitivity analysis for varying rates of demand, the retailers' carrying cost, the retailers' deterioration cost, the distributor's carrying cost, the suppliers' carrying cost and rates of deterioration are tabulated in Table 2 .

\section{Comment on the Sensitivity Analysis}

The main conclusions found from the sensitivity analysis are as bellows:

(1) When the retailers' demand rate increases, each player's total cost $T C_{r}, T C_{d}, T C_{p}$; the joint total cost $T C$ as well as the number of $n^{*}$; will increase also.

(2) When he distributor, the suppliers and the retailers' carrying cost increases, the number of $n^{*} 10$ remains the same, but the number of $n^{*} 01$ tends to decrease. If the carrying cost decreases, the reverse is also true.

(3) As the retailers' deterioration cost increases, the number of deliveries from distributor to retailers tends to increase. Each player's total cost, except the suppliers' cost tends to increase. The joint total cost $T C$ increases as well.

(4) As soon as the deterioration rateaincreases, each player's total cost and the joint total cost tend to increase. But, the number of deliveries remains the same.

(5) The joint total cost $T C$ is more sensitive to the parameter of the distributor's carrying cost $H_{d}$. The increase is over $20 \%$ as $H_{d}$ increases by $30 \%$.

Table 1.TC with different combinations of $n_{i k}$

\begin{tabular}{ccccccccc}
\hline$T$ & $n_{10}$ & $n_{01}$ & $n_{02}$ & $n_{03}$ & $T C_{r}$ & $T C_{d}$ & $T C_{p}$ & $T C^{*}$ \\
\hline 1 & 1 & 1 & 1 & 1 & 761007 & 20829 & $23219^{* *}$ & 805056 \\
1 & 1 & 5 & 5 & 23 & 125610 & 277077 & 23219 & 425906 \\
1 & 1 & 20 & 20 & 34 & 63605 & 284665 & 23219 & 371489 \\
$1^{*}$ & $1^{*}$ & $21^{*}$ & $21^{*}$ & $33^{*}$ & $63551^{*}$ & 284708 & 23219 & $371478^{*}$ \\
1 & 1 & 24 & 24 & 29 & 64150 & 284798 & 23219 & 372168 \\
1 & 2 & 1 & 1 & 1 & 379432 & $11832^{* *}$ & 665860 & 1057124 \\
1 & 2 & 1 & 1 & 22 & 280949 & 42021 & 665860 & 988830 \\
1 & 2 & 24 & 24 & 33 & 85106 & 142721 & 665860 & 893687 \\
\hline
\end{tabular}

Notes: * Optimal solution that minimizes TC.

** Optimal solution that minimizes $T C_{p}$ or $T C_{d}$.

Table 2. Sensitivity analysis when a parameter is changed.

\begin{tabular}{|c|c|c|c|c|c|c|c|c|c|}
\hline $\begin{array}{l}\text { Para- } \\
\text { meter }\end{array}$ & $\%$ changed & $n_{10}$ * & $n_{01}{ }^{*}$ & $n_{02}{ }^{*}$ & $n_{03}{ }^{*}$ & $T C_{r}$ & $T C_{d}$ & $T C_{p}$ & $T C\left(n_{i k}^{*}\right)$ \\
\hline \multirow{3}{*}{$d_{1}$} & No change & 1 & 21 & 21 & 33 & 63551 & 284708 & 23219 & 371478 \\
\hline & $-30 \%$ & 1 & 17 & 21 & 33 & 59460 & 251898 & 21694 & 333053 \\
\hline & $+30 \%$ & 1 & 23 & 21 & 33 & 67088 & 317484 & 24745 & 409317 \\
\hline \multirow{2}{*}{$H_{r 1}$} & $-30 \%$ & 1 & 23 & 21 & 33 & 59681 & 284589 & 23219 & 367489 \\
\hline & $+30 \%$ & 1 & 17 & 21 & 33 & 66925 & 284746 & 23219 & 374891 \\
\hline \multirow{2}{*}{$P_{r 1}$} & $-30 \%$ & 1 & 21 & 21 & 33 & 63389 & 284685 & 23220 & 371293 \\
\hline & $+30 \%$ & 1 & 20 & 21 & 33 & 63729 & 284708 & 23219 & 371657 \\
\hline \multirow{2}{*}{$H_{d}$} & $-30 \%$ & 1 & 20 & 21 & 33 & 63551 & 205544 & 23219 & 292314 \\
\hline & $+30 \%$ & 1 & 21 & 21 & 33 & 63603 & 363811 & 23219 & 450633 \\
\hline \multirow{2}{*}{$H_{p 1}$} & $-30 \%$ & 1 & 21 & 21 & 33 & 63551 & 284708 & 23219 & 371478 \\
\hline & $+30 \%$ & 1 & 21 & 21 & 33 & 63551 & 284708 & 23219 & 371478 \\
\hline \multirow{2}{*}{$\theta$} & $-30 \%$ & 1 & 21 & 21 & 33 & 63111 & 277315 & 19207 & 359633 \\
\hline & $+30 \%$ & 1 & 20 & 20 & 33 & 64024 & 292125 & 27272 & 383422 \\
\hline
\end{tabular}




\section{Conclusion}

This paper discusses the optimal joint-cost policy in a two-suppliers three-echelon inventory model that integrates the upper, middle, and lower levels of the supply chain. By using the integrated approach that takes account of the suppliers, the distributor and the retailers, the total joint cost is found to be less than the independent approach by the individual player. The result is validated through sensitivity analysis. Furthermore, recent advances in communications and information technology provide greater opportunity for significant savings in logistics cost by implementing strategic alliances within the supply chains.

\section{References}

1. Axsater, S., Continuous Review Policies for Multi-level Inventory System with Stochastic Demand in: Graves, S. C., Rinooy, K. A., and Zipkin P. (eds.), Handbook in Operations Research and Management Science, 4, 1993, pp. 175-197.

2. Bessler, S. A. and Veinott, A. J., Optimal Policy for a Multi-echelon Inventory Model, Naval Research Logistics Quarterly, 13, 1966, pp. 355-389.
3. Clark, A. J., and Scarf, H., Optimal Policy for a Mutli-echelon Inventory Problem, Management Science, 6, 1960, pp. 475-490.

4. Lin C., and Lin Y., A Cooperative Inventory Policy with Deteriorating Items for a TwoEchelon Model, European Journal of Operational Reseatch, 178, 2007, pp. 92-111.

5. Rau, H, Wu, M.Y., and Wee H.M., Integrated Inventory Model for Deteriorating Items under a Multi-Echelon Supply Chain Environment, International Journal of Production Economics, 86, 2003, pp. 155-168.

6. Rau, H, Wu, M. Y., and Wee, H. M., Deteriorating Item Inventory Model with Shortage due to Supplier in an Integrated Supply Chain, International Journal of System Science, 35, 2004, pp. 293-303.

7. Wee, H. M., Optimal Buyer-seller Discount Pricing and Ordering Policy for Deteriorating Items, The Engineering Economist, 43, 1998, pp. 151-168.

8. Yu, J. C. P., An Integrated Policy of Multi-Echelon Supply Chain with Dual Source for Deteriorating Items, Journal of Information and Optimization Sciences, 28, 2007, pp. 1-15. 